\title{
A Comparison between Vanadyl, Vanadate, and Decavanadate Effects in Actin Structure and Function: Combination of Several Spectroscopic Studies
}

\author{
S. Ramos, ${ }^{1,2}$ J. J. G. Moura, ${ }^{1}$ and M. Aureliano ${ }^{2}$ \\ ${ }^{1}$ REQUIMTE/CQFB, Departamento Química, Faculdade de Ciências e Tecnologia, \\ Universidade Nova de Lisboa, 2829-516 Caparica, Portugal \\ ${ }^{2}$ CCMar, DCBB, Faculdade de Ciências e Tecnologia, Universidade do Algarve, \\ 8005-139 Faro, Portugal
}

Correspondence should be addressed to M. Aureliano, maalves@ualg.pt

Copyright (C) 2012 S. Ramos et al. This is an open access article distributed under the Creative Commons Attribution License, which permits unrestricted use, distribution, and reproduction in any medium, provided the original work is properly cited.

\begin{abstract}
The studies about the interaction of actin with vanadium are seldom. In the present paper the effects of vanadyl, vanadate, and decavanadate in the actin structure and function were compared. Decavanadate clearly interacts with actin, as shown by ${ }^{51} \mathrm{~V}$-NMR spectroscopy. Decavanadate interaction with actin induces protein cysteine oxidation and vanadyl formation, being both prevented by the natural ligand of the protein, ATP. Monomeric actin (G-actin) titration with vanadyl, as analysed by EPR spectroscopy, indicates a $1: 1$ binding stoichiometry and a $k_{\mathrm{d}}$ of $7.5 \mu \mathrm{M}$. Both decavanadate and vanadyl inhibited G-actin polymerization into actin filaments (F-actin), with a $\mathrm{IC}_{50}$ of 68 and $300 \mu \mathrm{M}$, respectively, as analysed by light-scattering assays. However, only vanadyl induces G-actin intrinsic fluorescence quenching, which suggests the presence of vanadyl high-affinity actin-binding sites. Decavanadate increases (2.6-fold) actin hydrophobic surface, evaluated using the ANSA probe, whereas vanadyl decreases it (15\%). Finally, both vanadium species increased $\varepsilon$-ATP exchange rate $(k=6.5 \times$ $10^{-3}$ and $4.47 \times 10^{-3} \mathrm{~s}^{-1}$ for decavanadate and vanadyl, resp.). Putting it all together, it is suggested that actin, which is involved in many cellular processes, might be a potential target not only for decavanadate but above all for vanadyl.
\end{abstract}

Keywords: Actin, vanadyl, decavanadate, vanadate, cysteine oxidation, intrinsic fluorescence, ANSA probe, NMR, EPR

\section{Introduction}

Vanadium impact in biology, pharmacology, and medicine is well known, mainly after the discovery that the "muscle inhibitor factor", present in ATP obtained from horse muscle and responsible for $\mathrm{Na}^{+}, \mathrm{K}^{+}$-ATPase inhibition was, in fact, vanadate, which acts as a transition state analogue for phosphoenzyme hydrolysis, blocking the enzyme catalysis [1]. The most outstanding vanadium and vanadium compounds effect is perhaps the specific inhibition of protein tyrosine phosphatases (PTP), promoting an increasing of glucose uptake in several types of cells and mimicking insulin effects [2]. Additionally, the usage of vanadium as a tool to understand several biochemical processes is well 
recognized [3]. Actin is one of the most abundant proteins in cells, being involved in many cellular processes, such as muscle contraction, in muscular cells and cytoskeleton structure and dynamics, in nonmuscle cells. Very few studies concerning the interaction of vanadium with actin have been so far described [4-6]. These previous studies demonstrated that vanadate (vanadium (V)), upon binding to F-actin-ADP subunits, increases the strength of actin-actin interactions, stabilizing the F-actin filament [5], and vanadyl interaction with the monomeric G-actin reveals one strong binding site, among others [4]. More recently, it has been demonstrated that vanadate oligomers, such as tetrameric and decameric vanadate (decavanadate, $\mathrm{V}_{10}$ ), prevent $\mathrm{G}$-actin polymerization, whereas no effects were observed for vanadate up to $2 \mathrm{mM}$ [7]. It was also demonstrated that G-actin stabilizes decavanadate species, increasing its half-life time from 5 to $27 \mathrm{~h}$ [7]. On the other hand, cellular studies have shown that vanadium compounds induce changes in actin cytoskeleton, which are responsible for morphological and cell proliferation alterations [8,9]. These effects are probably induced through the inhibition of protein tyrosine phosphatases, as referred above, or eventually through reactive oxygen species generation, once it is well known that transition elements, such as vanadium, promote Fenton-like reactions. These actions could explain, at least in part, the antitumor effects of vanadium [10]. In the present paper, we compare the effects of vanadyl, vanadate, and decavanadate in actin structure and function, combining several spectroscopic and kinetic studies.

\section{Decavanadate, Vanadate, and Vanadyl Interactions with Actin: NMR and EPR Studies}

It was verified that decavanadate ${ }^{51} \mathrm{~V}$-NMR signals broadened upon actin titration, whereas no changes were observed for the other vanadate oligomers signals [11]. Decavanadate interactions with actin are of particular interest once it was observed that only $\mathrm{V}_{10}$ species are able to promote protein cysteine oxidation $[11,12]$. ATP prevents decavanadate reduction and protein cysteine oxidation $[11,12]$, suggesting interaction blockage, eventually due to the same protein-binding site. In fact, upon decavanadate interaction with actin, a concomitant reduction to vanadyl (V (IV)) was detected, as analysed by EPR spectroscopy (Figure 1). Typical EPR vanadium (IV) signals can be detected upon decavanadate incubation with actin (Figure 1(B)), as well as with vanadyl incubation with the protein (Figure 1(D)). The presence of ATP into the medium prevents decavanadate reduction to vanadyl (Figure 1(C)), as well as the interaction of vanadyl with actin (Figure 1(E)).

Recently reviewed decavanadate effects in biological systems have pointed out that decavanadate is more or less efficient than the corresponding simple oxovanadates in targeting specific proteins, particularly at the nucleotide-binding site [3, 13]. However, while the putative occurrence of decavanadate in cells remains to be completely elucidate, vanadate usually occurs inside the cells in its reduced form, vanadyl, being this cation recognized to bind to several proteins. We have shown recently, using EPR spectroscopy, that vanadyl interacts with G-actin with a dissociation constant $\left(k_{\mathrm{d}}\right)$ of $7.48 \pm 1.11 \mu \mathrm{M}$ and a $1: 1$ vanadyl-actin stoichiometry [11]. It was suggested that vanadyl binds to actin at the ATP-binding site, since the presence of this nucleotide in the medium assay prevents the interaction between the metal and the protein (Figure 1), as it was also observed for decavanadate $[11,12,14]$.

\section{Decavanadate, Vanadate, and Vanadyl Interactions with Actin: Fluorescence Studies}

As described above, decavanadate revealed a particular interaction with actin. As analysed by light scattering spectroscopy, it was observed that decavanadate prevents the extension of G-actin polymerization 


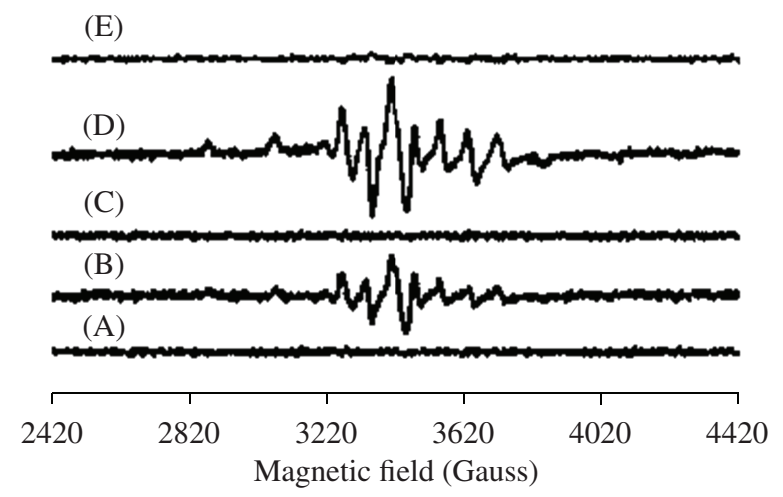

Figure 1: Frozen aqueous solution X-band EPR spectra. (A) $2 \mathrm{mM}$ Tris (pH 7.5), $0.2 \mathrm{mM} \mathrm{CaCl} 2$ and $25 \mathrm{mM}$ decavanadate with (B) $100 \mu \mathrm{M}$ G-actin, (C) $50 \mu \mathrm{M}$ G-actin plus $0.2 \mathrm{mM}$ ATP, (D) $2 \mathrm{mM}$ Tris (pH 7.5), $0.2 \mathrm{mM} \mathrm{CaCl}_{2}$, and $250 \mu \mathrm{M} \mathrm{VOSO}_{4}$ with $50 \mu \mathrm{M}$ G-actin, (E) same as (D) plus $0.2 \mathrm{mM}$ ATP. Vanadium (IV) EPR typical signals were observed upon both decavanadate (B) or $\mathrm{VOSO}_{4}$ (D) incubation with G-actin. No EPR signals were detected when ATP was present in the medium (C and E).

into F-actin filaments, being more effective than vanadyl [7, 14]. At the same experimental conditions, the observed $\mathrm{IC}_{50}$ for the inhibition of polymerization reaction was lower for decavanadate, by comparison with vanadyl (68 and $300 \mu \mathrm{M}$, resp.) whereas no effects were observed up to $2 \mathrm{mM}$ vanadate $[7,14]$. Further studies, using fluorescence spectroscopy, were performed to compare the interaction between decavanadate, vanadate, and vanadyl with actin. It was verified that vanadyl induces a total quenching of actin intrinsic fluorescence (Figure 2), whereas decavanadate increases its fluorescence $[12,14]$. It should be noted that decavanadate concentration used in these studies is 10 times less than that of vanadyl. This means that $500 \mu \mathrm{M}$ decavanadate (in total vanadate) corresponds to $50 \mu \mathrm{M}$ of decameric vanadate species. Regarding protein intrinsic fluorescence, care must be taken to evaluate the interaction of decavanadate or vanadate with proteins, due to inner filter effects [12]. However, many biochemical studies using vanadium do not take in consideration those effects, which decreases the fluorescence measurements accuracy. For a decavanadate solution with a concentration as lower as $0.05 \mathrm{mM}$ (meaning $0.5 \mathrm{mM}$ total vanadium), the absorbance values at excitation and emission wavelengths used in assays (295 and $340 \mathrm{~nm}$, resp.) attain to $1.72 \mathrm{OD}(1.17+0.55)$, which is more than vanadate $(1.02 ; 0.85+0.17)$ or vanadyl $(0.17 ; 0.13+0.04)$, for the same vanadium concentrations. Correction of the fluorescence intensities due to these inner filter effects is desirable and can be done using proper equations [15].

Besides the effects on intrinsic fluorescence, it was also observed that actin hydrophobic surface, as determined using the ANSA probe, increases upon decavanadate exposure (2.6-fold), whereas vanadyl promotes its decreasing by $15 \%$, suggesting that the changes caused by the former are clearly different from the ones induced by vanadyl, favouring a protein hydrophobic environment $[12,14]$. However, both decavanadate and vanadyl (up to $200 \mu \mathrm{M}$ total vanadium) increased $\varepsilon$-ATP exchange rate $\left(k=6.5 \times 10^{-3}\right.$ and $4.47 \times 10^{-3} \mathrm{~s}^{-1}$, resp., in comparison with the control: $\left.k=3.0 \times 10^{-3} \mathrm{~s}^{-1}\right)$, and both species decreased ATP exchange half-life time, denoting a more available cleft [12, 14]. Actin conformational changes induced by vanadyl were also verified by ${ }^{1} \mathrm{H}-\mathrm{NMR}$ [14]. 


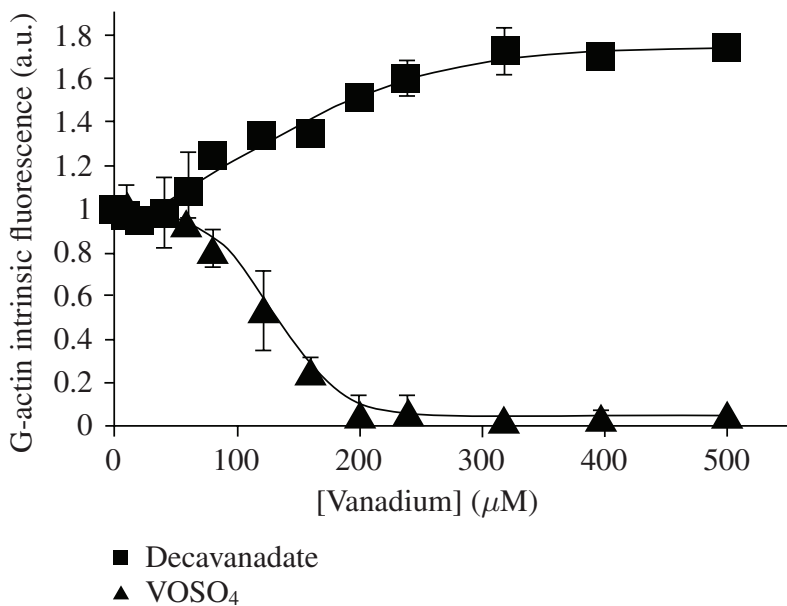

Figure 2: G-actin intrinsic fluorescence. G-actin $(5 \mu \mathrm{M})$ was incubated with $\mathrm{VOSO}_{4}(\boldsymbol{\Delta})$ or decavanadate (ם) for $20 \mathrm{~min}$, at $25^{\circ} \mathrm{C}$, in $2 \mathrm{mM}$ Tris- $\mathrm{HCl}(\mathrm{pH} 7.5), 0.2 \mathrm{mM} \mathrm{CaCl}_{2}$. The maximum of intrinsic fluorescence spectra $\left(\lambda_{\mathrm{ex}}=295 \mathrm{~nm}\right)$ were plotted against vanadyl or decavanadate (total) concentrations, considering 1.00 the value for native actin. The results shown are the average of, at least, triplicated measurements.

\section{Concluding Remarks}

In order to evaluate and to compare the effects in actin structure and function, a major and relevant cellular protein, induced by several vanadium species, namely, vanadyl, vanadate, and decavanadate, a combination of EPR, ${ }^{51} \mathrm{~V}$-NMR and fluorescence spectroscopic studies along with kinetic ones was used. Taken together, the studies reveal the presence of G-actin high-affinity-binding sites for vanadyl, with a 1:1 actin-vanadium (IV) stoichiometry. Also, a specific decavanadate interaction with the protein was observed, leading to cysteine oxidation and vanadyl formation. Both vanadium species interactions with actin were prevented by ATP. Putting it all together, it is proposed that the biological effects of vanadium, which major biological role still to be clarified, may be explained, at least in part, by its capacity to interact with actin and to affect several biological processes where actin may be involved. It is concluded in this paper that (i) decavanadate and vanadyl inhibit actin polymerization, at $\mu \mathrm{M}$ concentrations; (ii) only decavanadate interaction with actin induces cysteine oxidation and vanadate reduction, being these effects are prevented by ATP; (iii) decavanadate and vanadyl induce actin conformational changes affecting protein ATP-binding site; (iv) the presence of actin high-affinitybinding sites for vanadyl. It is suggested that actin, a protein involved in many cellular processes, is a plausible protein target for decavanadate and above all for vanadyl.

\section{Abbreviations}

ANSA: 8-anilino-1-naphthalene sulfonic acid

G-actin: Monomeric actin

F-actin: Filamentous polymerized actin. 


\section{Acknowledgments}

This work was supported by CCMAR funding. S. Ramos would like to thank to the Portuguese Foundation "Fundação para a Ciência e Tecnologia (FCT)" for the PhD Grant SFRH/BD/29712/2006.

\section{References}

[1] L. C. Cantley Jr., L. Josephson, and R. Warner, "Vanadate is a potent (Na,K)-ATPase inhibitor found in ATP derived from muscle," Journal of Biological Chemistry, vol. 252, no. 21, pp. 7421-7423, 1977.

[2] E. Tsiani, E. Bogdanovic, A. Sorisky, L. Nagy, and I. G. Fantus, "Tyrosine phosphatase inhibitors, vanadate and pervanadate, stimulate glucose transport and GLUT translocation in muscle cells by a mechanism independent of phosphatidylinositol 3-kinase and protein kinase C," Diabetes, vol. 47, no. 11, pp. 1676-1686, 1998.

[3] M. Aureliano and D. C. Crans, "Decavanadate $\left(\mathrm{V}_{10} \mathrm{O}_{28}\right)$ and oxovanadates: oxometalates with many biological activities," Journal of Inorganic Biochemistry, vol. 103, no. 4, pp. 536-546, 2009.

[4] F. An, B.-Y. Zhang, B.-W. Chen, and K. Wang, "The interaction of vanadyl ions with G-actin," Chemical Journal of Chinese Universities, vol. 17, no. 5, pp. 667-671, 1996.

[5] C. Combeau and M. F. Carlier, "Probing the mechanism of ATP hydrolysis on F-actin using vanadate and the structural analogs of phosphate BeF-3 and A1F-4.," Journal of Biological Chemistry, vol. 263, no. 33, pp. 17429-17436, 1988.

[6] S. C. El-Saleh and P. Johnson, "Non-covalent binding of phosphate ions by striated muscle actin," International Journal of Biological Macromolecules, vol. 4, no. 7, pp. 430-432, 1982.

[7] S. Ramos, M. Manuel, T. Tiago et al., "Decavanadate interactions with actin: inhibition of G-actin polymerization and stabilization of decameric vanadate," Journal of Inorganic Biochemistry, vol. 100, no. 11, pp. 1734-1743, 2006.

[8] J. Rivadeneira, D. A. Barrio, G. Arrambide, D. Gambino, L. Bruzzone, and S. B. Etcheverry, "Biological effects of a complex of vanadium(V) with salicylaldehyde semicarbazone in osteoblasts in culture: mechanism of action," Journal of Inorganic Biochemistry, vol. 103, no. 4, pp. 633-642, 2009.

[9] X. G. Yang, X. D. Yang, L. Yuan, K. Wang, and D. C. Crans, "The permeability and cytotoxicity of insulin-mimetic vanadium compounds," Pharmaceutical Research, vol. 21, no. 6, pp. 1026-1033, 2004.

[10] A. M. Evangelou, "Vanadium in cancer treatment," Critical Reviews in Oncology/Hematology, vol. 42, no. 3, pp. 249-265, 2002.

[11] S. Ramos, R. O. Duarte, J. J. G. Moura, and M. Aureliano, "Decavanadate interactions with actin: cysteine oxidation and vanadyl formation," Dalton Transactions, no. 38, pp. 7985-7994, 2009.

[12] S. Ramos, J. J. G. Moura, and M. Aureliano, "Actin as a potential target for decavanadate," Journal of Inorganic Biochemistry, vol. 104, no. 12, pp. 1234-1239, 2010.

[13] M. Aureliano, "Decavanadate: a journey in a search of a role," Dalton Transactions, no. 42, pp. 9093-9100, 2009.

[14] S. Ramos, R. M. Almeida, J. J. G. Moura, and M. Aureliano, "Implications of oxidovanadium(IV) binding to actin," Journal of Inorganic Biochemistry, vol. 105, no. 6, pp. 777-783, 2011.

[15] J. R. Lackowicz, Principles of Fluorescence Spectroscopy, Plenum Press, New York, NY, USA, 1983. 


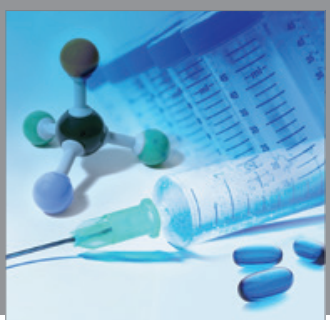

International Journal of

Medicinal Chemistry

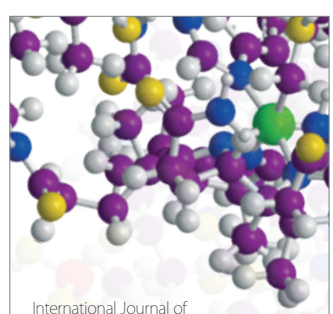

Carbohydrate Chemistry

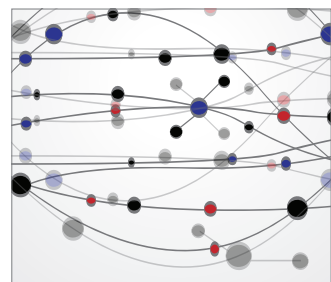

The Scientific World Journal
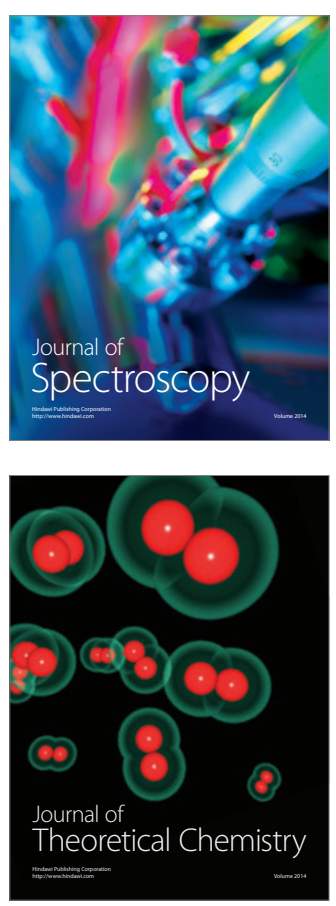
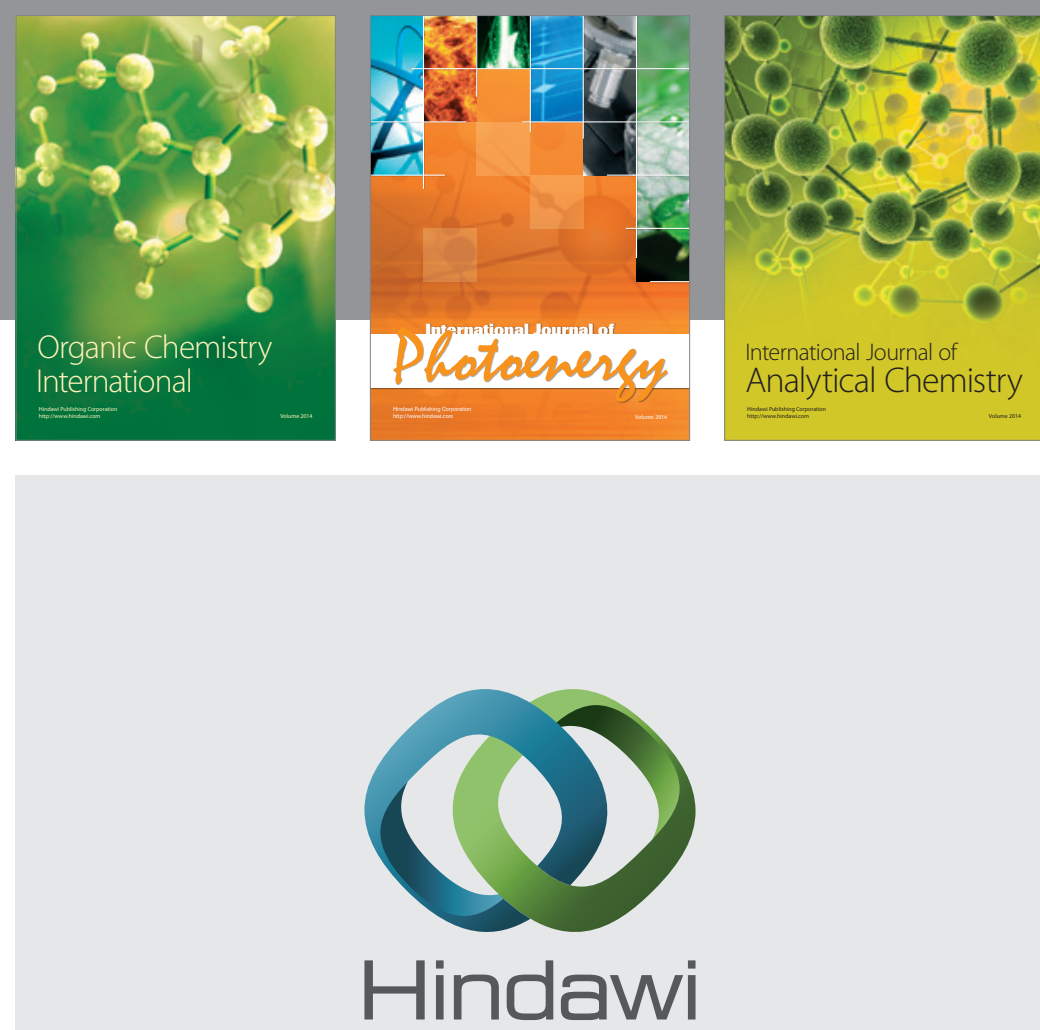

Submit your manuscripts at

http://www.hindawi.com
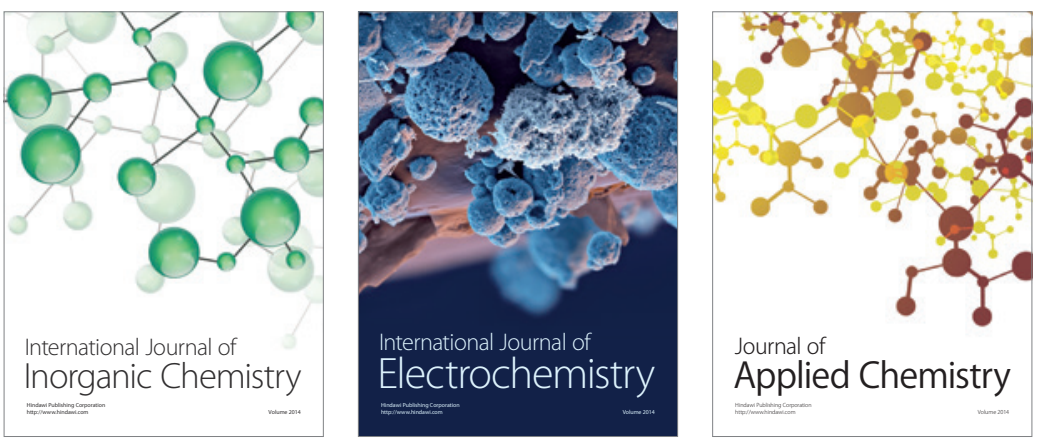

Journal of

Applied Chemistry
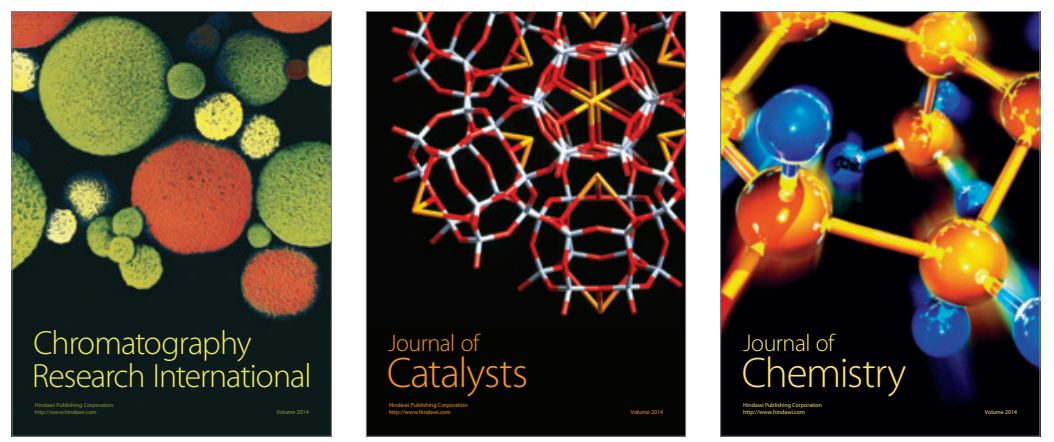
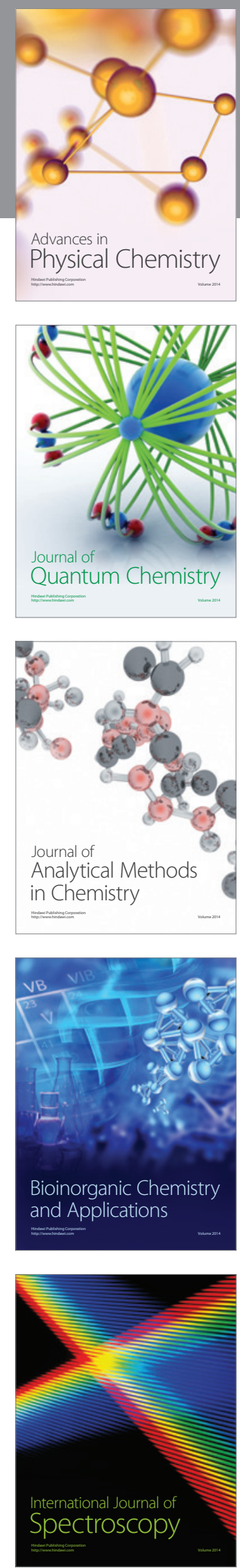